\title{
Virtual Reality approaches for evacuation simulation of various disasters
}

\author{
Naohiro Takeichi ${ }^{1}$, Takeshi Katagiri ${ }^{2}$, Harumi Yoneda ${ }^{3}$, Shusaku Inoue ${ }^{4}$, \\ Yusuke Shintani ${ }^{5}$ \\ ${ }^{1}$ Advanced Design Department/Takenaka Corporation, Tokyo, Japan \\ takeichi.naohiro@takenaka.co.jp \\ 2,3,4,5 Research and Development Institute/Takenaka Corporation, Chiba, Japan \\ katagiri.takeshi@takenaka.co.jp; nishimura.harumi@takenaka.co.jp; inoue.shuusaku@takenaka.co.jp; \\ shintani.yuusuke@takenaka.co.jp
}

\begin{abstract}
This paper presents a virtual reality (VR) system of evacuation in the cases of various disasters. We considered a combination of disasters under realistic scenarios. Disaster simulations by numerical models were imported into the VR system to express a realistic situation. Not only disaster experts but also designers and non-professionals are able to share realistic experiences for escape from the disasters. This system is useful for performance-based design, planning of escape, disaster prevention, evacuation drill etc. The VR system consists of Building Information Modelling, physical simulation models for disasters and human behaviour's simulation.
\end{abstract}

Keywords: virtual reality, multi-agent dynamics, performance-based design, serious gaming, CFD, BIM

\section{Introduction}

It is difficult for usual people to imagine real situations in buildings under disasters like earthquake, tsunami, storm tide, fire, etc. Because of that, they often delayed in starting evacuation in emergencies. For example, in case of tsunami after a big earthquake, people have to go to high ground or upper floors when they feel ground shaking. In other hand, people immediately move downstairs to go outside in case of fire. How to escape from disasters depends on types of them. Habitants and crowd handlers have to judge the best way to evacuate in an emergency. Planners, designers and engineers have to design buildings to support them. However those experts also lack knowledge and experience of the disasters.

To help them, various disasters simulations and their evacuation models have been developed so far. Although each single hazard or human behaviour have been studied, but simulations for complex of hazards and their evacuation are still insufficient to let designers imagine realistic situations. In actual disasters, several hazards happen in same time and people have to consider them.

To solve the problem, we have tried to integrate disasters and evacuation simulations, and visualize the results on single VR system, named "maXim". [1]

\section{Contents of Virtual reality}

Building data is based on Building Information Model (BIM). Modelling approaches of simulations are based on as below:

Human behaviour: multi-agent dynamics

Seismic response: real-time physical dynamics of gaming technology

Tsunami: $\quad$ Flood dynamics by Navier-Stokes with Volume of Fluid Methods

Fire \& smoke: $\quad$ Computational Fluid Dynamics (CFD)

Under scenarios assuming disasters, hazards and human behaviours are simulated by each simulator and the logs of simulations are installed into the virtual space. The VR can be expressed by PC monitor, head mount display, and dome theatre. (Fig. 1.)

For convenience of the paper, only the outline of each simulation will be described. 


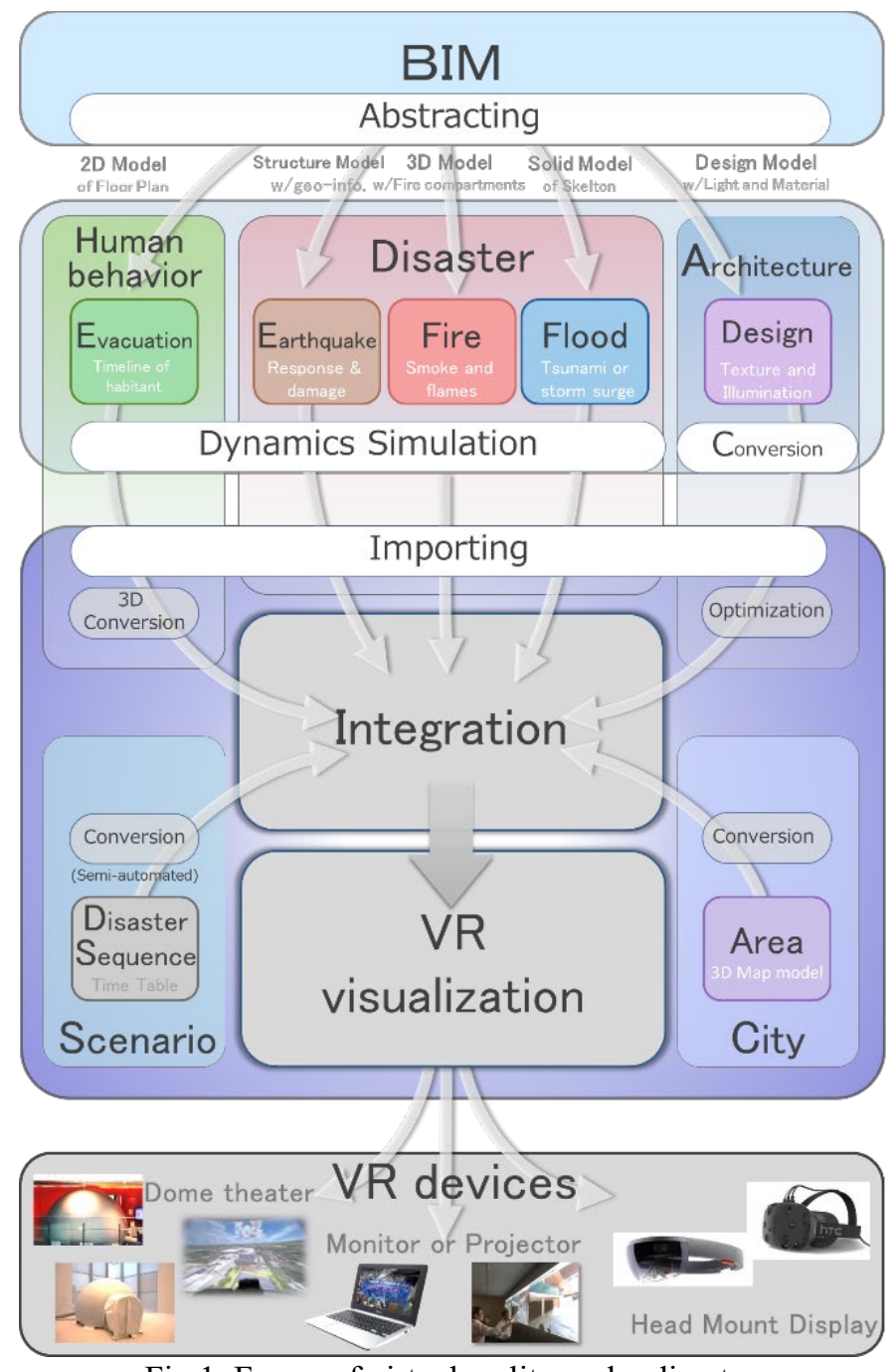

Fig.1. Frame of virtual reality under disasters

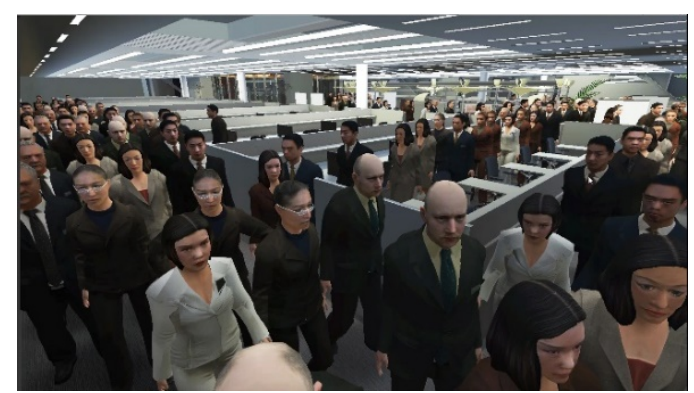

Fig. 2. Scene of evacuation in an office building.

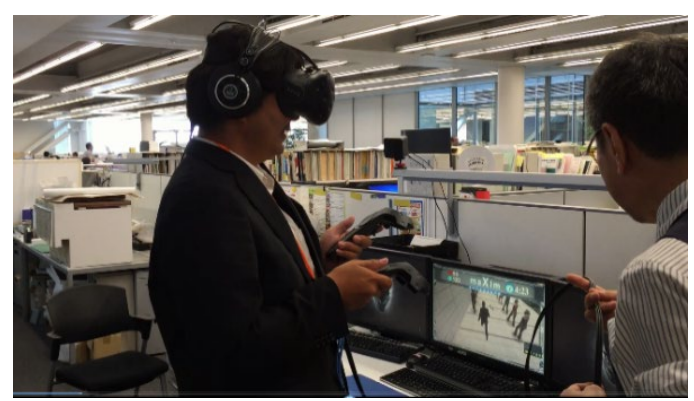

Fig. 3. Virtual evacuation drill

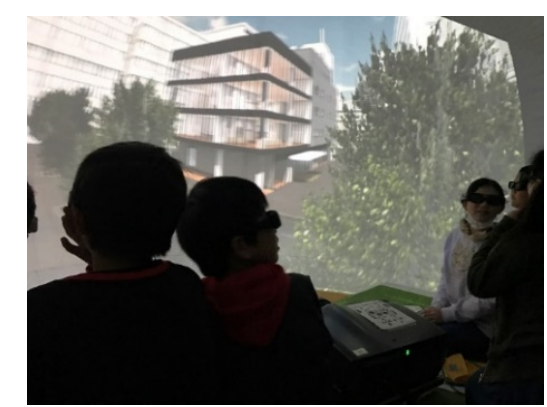

Fig. 4. Virtual reality in the dome theatre

\subsection{Human behaviour - multi-agent simulation}

Multi-agent simulation "SimTread" has been developed as a pedestrian simulation system following a straightforward, highly user-definable pedestrian and spatial model. Unlike network and mesh models, this pedestrian model is a multi-agent system, where an individual pedestrian is modelled as an agent, and the spatial model is based on actual coordinates; this enables the direct representation of plans.[2]

The crowd characteristics represented by this system have been evaluated through test cases by measuring the flow rate, a primary indicator of crowd evaluation [3][4].

\subsection{Seismic response}

The behaviours of furniture during earthquake are calculated with real-time physical dynamics of gaming technology by using physics engines named physX in Unity. Where, the floor response that is obtained by simulation with another structural model is imported.

We modelled furniture as rigid bodies attached colliders and joint constraints. We gave the values of weight and centre of mass and material properties such as coefficients of friction and bounciness to each model. Since material properties are given to each object not between the objects, the selection of the combine function affects simulation [5]. As phys X is a commercial engine, the implementation details are unknown. We determined these values by reference to simulations and experiments that we conducted. 


\subsection{TSUNAMI - numerical methodology}

Flow-3D is a general-purpose CFD package that is used to solve transient and three dimensional flow problems. It was developed by Flow Science, Inc., and has a comprehensive track record in CFD modelling. Though, the application in modelling tsunami on building is a new attempt. Flow-3D is developed based on the fractional volume of fluid (VOF) free surface tracking method. Under this method, cells are defined with a value between zero and one for empty to fully filled cells with fluid. For partially filled cells, the slope of the free surface is determined by an algorithm that uses the surrounding cells to define a surface angle and a surface location. This method allows the steep fluid slopes to be defined and it is applicable to describe the breaking wave in tsunami.

The model used in this research is an incompressible and viscous flow model. Flow-3D employs the finite difference method to solve the fluid equations of motion. The computational domain is defined in a fixed rectangular grid [6].

\subsection{Fire \& Smoke - FDS}

Fire and smoke simulation was conducted by using Fire Dynamics Simulator (FDS) [7] developed by National Institute of Standards and Technology (NIST). FDS solves numerically a large eddy simulation form of the Navier-Stokes equations with an emphasis on smoke and heat transport from fires.

The 3D model of the rooms was imported from the BIM data automatically using third party pre-processing tool of FDS. The calculation conditions such as mesh, material properties, and boundary conditions etc. were set into the model. The fire source was also set in the model based on the fire scenario. The fire behaviour such as smoke movement and flame shapes were calculated. Virtual Reality System imported the calculation results such the temperature, the velocity, the transmission in each cell as at each time step.

\section{Conclusions}

The VR system can simulate earthquakes, tsunamis, fires and evacuation from them under realistic scenario by PC monitor, head mount display, and dome theatre.

Not only researchers and professionals, but also ordinary citizens without technical knowledge can experience virtual escape from the hazards that is useful for performance-based design, planning of escape, disaster prevention, evacuation drill etc. (Fig. 3, 4.)

\section{References}

[1] Sample movies: https://www.youtube.com/watch?v=Vm7rFCUCYuk https://www.youtube.com/watch?v=iURFNCBbJy4

[2] T. Kimura, N. Takeichi, et al; Representing crowds using a multi-agent model-Development of the SimTread pedestrian simulation system, Japan Architectural Review, volume 2, pp.101-110, Jan. 2019

[3] Tomonori Sano, Yoshiyuki Yoshida, Naohiro Takeichi, Takeshi Kimura, Yoshikazu Minegishi; Experimental Study of Crowd Flow Passing through Simple-shaped Room and Validation for an Evacuation Simulator, Pedestrian and Evacuation Dynamics 2011p.587 - 599, 2011

[4] Akihide Jo, et al; Analysis of crowd flow capacity through a door connected to a crowded corridor., The Conference on Pedestrian and Evacuation Dynamics 2014 (PED2014), Transportation Research Procedia, vol.2, pp.10-18, 2014.10

[5] Unity: Unity User Manual (2018.1)/ Physics/3D Physics Reference/ Physic Material/ https://docs.unity3d.com/2018.1/Documentation/Manual/class-PhysicMaterial.html (2018.5.17)

[6] Shusaku Inoue and Yasunori Mizushima; Simulation of three-dimensional Tsunami inundation into the inside of a building, 10th International Conference on Urban Earthquake Engineering, March 1-2, 2013, Tokyo Institute of Technology, Tokyo, Japan

[7] K. McGrattan, et al, Fire Dynamics Simulator Technical Reference Guide Volume 1: Mathematical Model, NIST Special Publication, 2017 